\title{
LA EVALUACIÓN DE PROYECTOS DE INVERSIÓN MEDIANTE OPCIONES REALES: ASPECTOS CONCEPTUALES
}

\author{
Diego Fernando Manotas Duque* \\ Pablo César Manyoma Velásquez**
}

* Ingeniero Industrial - Especialista en Finanzas Universidad del Valle.

Profesor Asistente - Escuela de Ingeniería Industrial y Estadística - Facultad de Ingeniería Universidad del Valle.

** Ingeniero Industrial - Especialista en Sistemas Gerenciales de Ingeniería - Pontificia Universidad Javeriana.

Profesor Auxiliar - Escuela de Ingeniería Industrial y Estadística - Facultad de Ingeniería - Universidad delValle.

\section{RESUMEN}

El presente artículo ilustra los principales aspectos conceptuales que soportan el análisis económico de proyectos de inversión mediante opciones reales. Esta metodología se constituye en una forma diferente de pensar en ingeniería económica. En el artículo se ilustran aspectos como opciones financieras, opciones reales, valoración de opciones y la analogía entre opciones de compra y proyectos de inversión.

\section{ABSTRACT}

This paper introduces the main concepts that give support to the economic analysis of invesment 
projects using real options. This methodology is a different way of thinking in engineering economy. The paper shows topics as financial options, real options, options assessment and the analogy between call options and capital invesment projects.

\section{INTRODUCCIÓN}

El principal objetivo de la evaluación económica y financiera de proyectos de inversión, es el desarrollo de técnicas, modelos y criterios que permitan analizar de la mejor manera posible, la viabilidad de realizar un proyecto, considerando como premisa básica la necesidad de incrementar la riqueza del inversionista que toma decisiones frente al proyecto. Históricamente, los inversionistas han acudido a un cierto número de técnicas de reconocida aceptación, pero que como modelos que son, están fundamentados en algunos supuestos que no en todas las oportunidades se cumplen. Es preciso entonces conocer las nuevas tendencias en cuanto a técnicas y modelos de evaluación de proyectos se refiere.

Los criterios o modelos de decisión que se estudian en las universidades, particularmente en los cursos de Ingeniería Económica, se desarrollan en torno a proyectos cuyos elementos (inversiones, ingresos, egresos y valores de mercado) se asumen como magnitudes con una total certeza de ocurrencia, cuando la realidad nos enfrenta a situaciones en las que prima el riesgo y en otras ocasiones la incertidumbre.

Mo obstante, es conveniente anotar que en algunas Universidades se ofrecen cursos que corrigen los problemas asociados al "determinismo" de la Ingeniería Económica. Estos cursos giran en torno al análisis de riesgo y son más frecuentes a nivel de ciertos programas de posgrado. En términos generales, estos cursos incorporan aspectos como análisis de sensibilidad, el criterio de valor esperado, árboles de decisión y técnicas de simulación como Montecarlo.
Para refrendar estas afirmaciones, basta sólo con mirar cuáles son los proyectos que se evalúan actualmente. Es indudable que la tendencia ha cambiado. Las empresas de hoy realizan una gran cantidad de inversiones que parecen ser motivadas por decisiones que no consideran como elemento fundamental el factor económico. ¿Pero, será esto razonable en la práctica?. De hecho no, el objetivo implícito o explícito detrás de cualquier decisión en la vida empresarial se circunscribe al impacto económico, medido mediante la generación de riqueza para los inversionistas. Los modelos de decisión estudiados en ingeniería económica se centran en el análisis de flujos de caja y la determinación de criterios como el valor presente neto (VPM), la tasa interna de retorno (TIR), la tasa interna de retorno modificada (TIRM), por mencionar algunos, pero al parecer estos criterios no son suficientes al momento de evaluar inversiones en sistemas de información, programas de calidad, programas de mejoramiento continuo, programas de investigación y desarrollo, alianzas estratégicas, joint ventures, etc. La razón de esta insuficiencia, es la incertidumbre que producen proyectos cuyo desarrollo se ve condicionado por los acontecimientos que se presentan durante la misma etapa de evaluación. La nueva realidad empresarial nos enfrenta a proyectos con un alto nivel de incertidumbre, grandes necesidades de capital, complejas estructuras de activos y una severa presión de los sistemas financieros y en general económicos. Cada vez es más complejo cuantificar económicamente los beneficios derivados de un proyecto de inversión.

Por las anteriores razones, se ha venido gestando una nueva tendencia en la planeación financiera empresarial conocida como la Teoría de Opciones o también denominada Análisis de Opciones Reales, término acuñado por Stewart Myers profesor de la Escuela de Megocios MIT. El trabajo

1 Sloan School of Management Massachusest Institute of Technology 
en torno al análisis de opciones reales, es un tema de candente actualidad en materia de investigación en las más prestigiosas universidades del mundo. De hecho, en los últimos tres años las principales publicaciones de investigación financiera en Europa y Estados Unidos incorporan diferentes trabajos doctorales en relación con la técnica de análisis de opciones. Sin lugar a dudas uno de los catalizadores de esta gran revolución académica ha sido la gran aceptación de la teoría de valoración de opciones financieras impulsada por Fisher Black, Robert Merton y Myron Scholes. Sobre este trabajo se hará una referencia más adelante.

Considerando la poca difusión que estos trabajos han tenido a nivel latinoamericano y nacional particularmente, los autores del presente artículo pretenden mostrar las bases conceptuales que fundamentan el análisis de proyectos de inversión mediante opciones reales. Los autores ven en esta metodología, un gran potencial de investigación y aplicación de cara a mejorar los procesos de toma de decisiones a nivel empresarial, punto clave de la investigación en ingeniería.

El análisis de opciones reales está fundamentado en la premisa simple de que las decisiones de invertir o de no hacerlo son opciones o caminos alternativos que deben ser evaluados en un proyecto. Este análisis no es sustituto del análisis tradicional de flujos de caja, por el contrario contribuye a entender y obtener un mejor provecho de la incertidumbre, tratando de valorar las múltiples alternativas que seguramente presentará en el futuro cualquier proyecto.

La no consideración de las opciones implícitas en un proyecto conlleva a subvalorarlo y en general a desechar proyectos que se deberían realizar. De forma análoga si un proyecto implica opciones que podrían ejercer terceros, la flexibilidad futura del mismo podría tener impactos negativos sobre el desempeño financiero del proyecto.

\section{Qué son las opciones:}

La literatura financiera define el término "opciones" como un instrumento financiero perteneciente al grupo de los denominados derivativos, del cual hacen parte también los futuros y los acuerdos de permuta financiera, más conocidos como swaps. De esta manera, una opción puede definirse como un instrumento financiero que concede el derecho, no la obligación de comprar o vender un cierto activo a un precio determinado en una fecha futura. Para adquirir este derecho es necesario pagar una prima si es comprador de opciones o recibir una prima si es vendedor de opciones.

Las opciones de venta (Put Options) conceden al comprador el derecho, pero no la obligación, de vender un determinado activo a un determinado precio y en una fecha establecida. El vendedor de la opción de venta tiene la obligación de comprar el activo en la fecha acordada y al precio acordado si el comprador decide ejercer la opción.

Por otro lado, las opciones de compra (Call Options) son contratos por los cuales el comprador tiene el derecho pero no la obligación de comprar un determinado activo subyacente, a un determinado precio o precio de ejercicio y en una determinada fecha de vencimiento. El vendedor o suscriptor tiene la obligación de vender el activo subyacente en la fecha determinada y al precio acordado si el comprador ha decidido ejercer la opción.

Cuando se habla de opciones financieras es posible definir cuatro operaciones básicas que se describen a continuación:

- Comprar una opción de compra (buy a call)

- Vender una opción de compra (write a call)

- Comprar una opción de venta (buy a put)

- Vender una opción de venta (write a put)

Implícitamente cuando se analiza un proyecto de inversión se hace una analogía del mismo con un 
portafolio de bonos o títulos de deuda sin riesgo, de esta manera se visualiza el proyecto como una serie de flujos positivos o negativos que simplemente sucederán en el futuro, posterior a la realización de una serie de inversiones. El planteamiento básico, detrás de la teoría de opciones, conduce a pensar que esta analogía no es del todo cierta, y por lo tanto, la evaluación de un proyecto de inversión debe depender del tipo de proyecto analizado. Cuando se analizan proyectos de explotación de recursos naturales, proyectos de investigación y desarrollo o proyectos de sistemas de información por mencionar algunos ejemplos, parece ser que las opciones financieras y particularmente las opciones de compra (tipo call) resultan ser una mejor analogía que los bonos, considerando que en dichos proyectos los inversionistas siempre tienen opciones o caminos alternativos frente a los acontecimientos que se van presentando en el proyecto.

Para entender el concepto de opción de compra (call option) se hará uso de un ejemplo:

Analice la siguiente opción Call sobre libras esterlinas (activo subyacente). La opción le da el derecho a comprar $25.000 £$ en un plazo de dos meses y a un precio de ejercicio (strike-price) de $1.8 U \$ / £$. El costo de la opción (prima) será de 0.04 US\$/E.

La figura 1 y la tabla anexa permiten ilustrar, cuál es el potencial de utilidad o pérdida que puede tener un inversionista en una opción como la que se propone en el ejemplo.

Si durante el período de vigencia de la opción el precio de la libra esterlina se encuentra por debajo del precio de ejercicio (strike-price) estipulado en la opción (1.8 US\$/£), se deduce que un inversionista racional no ejercería la opción de compra, por lo tanto la pérdida máxima en la que incurriría estaría dada por el costo de la prima, es decir:

Costo de la prima (costo de ejercer opción): $25.000 £ \times 0,04$ US\$/£ $=1.000$ US\$
En la tabla anexa a la gráfica, se puede observar que para un precio de la libra esterlina de 1.84 US\$/£, se obtiene el punto de equilibrio del comprador de la opción, es decir, el flujo de caja neto es igual a cero.

De igual manera se puede ver que el potencial de utilidad de una opción tipo call ocurre cuando el precio del activo subyacente al momento de ejercer la opción es superior al precio de ejercicio más el valor de la prima por ejercer la opción.

Cuando el precio del activo subyacente es igual al precio de ejercicio se dice que la opción se encuentra "at the money", cuando se encuentra por debajo se dice que la opción está "out the money" y cuando se encuentra por encima se dice que está "in the money".

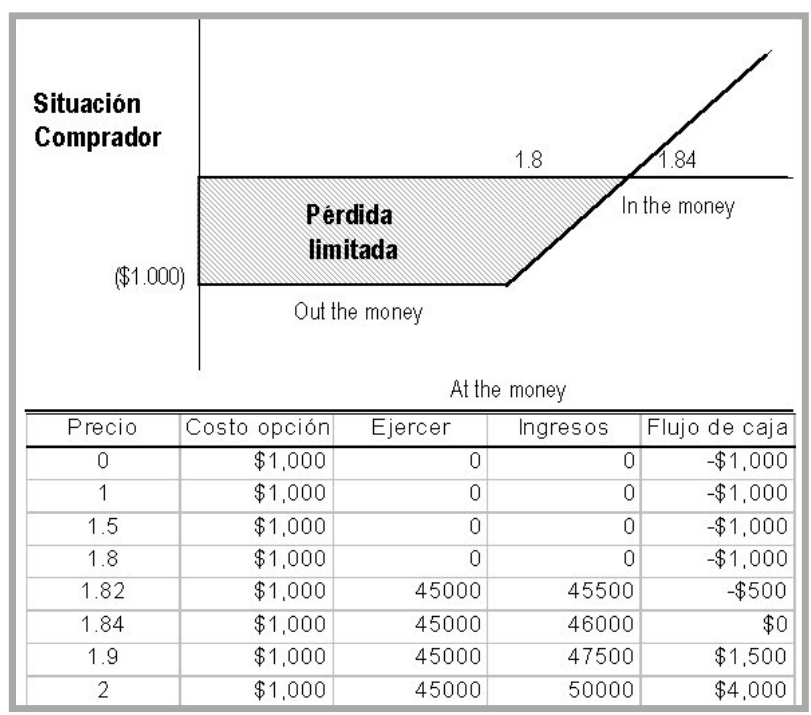

Figura 1. Opción de compra (Call option)

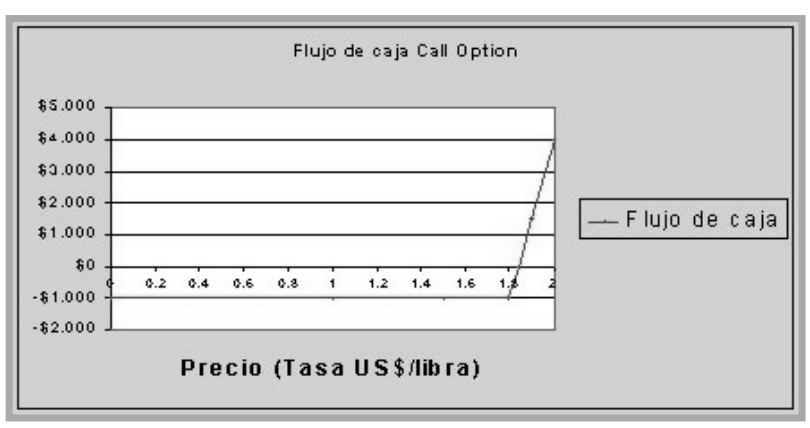

Figura 2. Perfil de riesgo de una opción (Call option) 
Del análisis de las figuras anteriores se puede deducir que cuando se invierte en opciones financieras tipo call se tiene la posibilidad de ejercer o no ejercer la opción dependiendo del comportamiento del precio del activo subyacente, en el caso del ejemplo, del comportamiento del precio de la libra esterlina $(f)$.

\section{¿Qué son las opciones reales?}

En un sentido estricto, algunos autores definen la metodología de opciones reales como una extensión de la teoría de opciones financieras a las opciones sobre activos reales (no financieros). La diferencia fundamental entre las opciones financieras y las reales, es que las primeras se detallan en un contrato, mientras que las segundas deben ser identificadas y especificadas en el marco de inversiones estratégicas. Autores como Stewart Myers, profesor de MIT consideran que el análisis de opciones reales es el eslabón que hace falta entre la planeación estratégica y las finanzas.

Las opciones son decisiones contingentes, es decir, que su adopción depende del curso de los acontecimientos. Frente a un proyecto, se toma una decisión al momento de invertir, si el proyecto es considerado viable, pero si el desempeño financiero no es el esperado, es muy posible que se adopte otra alternativa. Esta situación nos conduce a pensar que es un error considerar que el retorno de una opción sea lineal, de hecho, cambia en función de la decisión. Al contrario de las opciones, algunas decisiones no son modificables, por lo que se puede afirmar que su retorno es completamente lineal, independientemente de los sucesos que ocurran, en otras palabras, suceda lo que suceda la decisión siempre es la misma.

A manera de ilustración, los principales tipos de opciones reales identificados hasta el momento en trabajos de investigación previos son:

2 Real Options Managing Strategic Invesment in a Uncertain World. Martha Amran - Malin Kulatilaka
- Opciones de aplazamiento.

- Opciones de diferir las inversiones iniciales por etapas.

- Opciones de expansión de la capacidad de operación.

- Opción de reducción de la capacidad de operación.

- Opción de cierre de la capacidad de operación

- Opciones de abandono.

- Opciones de crecimiento.

- Opciones complejas con múltiples iteraciones.

\section{Valoración de opciones - La teoría que logró el Premio Mobel de Economía:}

Comprendidas las opciones tipo call, el paso siguiente es determinar los factores que condicionan el valor de una opción de este tipo. Las técnicas de valoración de opciones financieras son uno de los temas matemáticamente más complejos en finanzas y su discusión bien podría ser tema de otro artículo. Por este motivo se hará una breve referencia a uno de los trabajos de investigación más importantes desarrollados en el ámbito de las finanzas corporativas, el famoso modelo de Black $\&$ Scholes. Este modelo ha sido ampliamente discutido en la literatura financiera y a través de él es posible intentar una valoración de proyectos de inversión analizados como opciones reales, aunque este no sea el objetivo principal para el cual fue construido el modelo. Además del modelo de Black \& Scholes, se podrían mencionar los llamados métodos binomiales de valoración, que no son objeto de estudio de este artículo. Mo sobra aclarar que algunos autores consideran que el modelo de Black \& Scholes, si bien, logró interesantes resultados en el ámbito de las opciones financieras, tiene algunos problemas al momento de utilizarse para valorar opciones reales. Aparece aquí uno de los primeros polos de investigación financiera: EI desarrollo de modelos de valoración de opciones reales.

El desarrollo de Fischer Black, Myron Scholes y Robert Merton es un modelo matemático continuo que permite estimar el valor de una opción sobre acciones, basándose en una serie de factores y supuestos: 
- Las acciones (activo subyacente) no pagan dividendos durante el período de maduración de la opción.

- Las opciones se ejercen al vencimiento (opciones europeas).

- Los mercados financieros son eficientes.

- Mo existen comisiones ni gastos de transacción.

- Las tasas de interés se consideran conocidas y constantes durante el período de valoración.

- Los retornos están sujetos a una distribución lognormal.

La solución desarrollada por Black, Merton y Scholes fue una desviación radical del análisis de flujo de caja descontado. Los tres se basaban en los factores que podrían alterar el valor de una opción financiera a lo largo del tiempo. ¿Cuál es el valor de una opción al momento de adquirirla? ¿Cuál es el valor de dicha opción, cierto tiempo después, cuando el valor del activo subyacente (acción) ha cambiado?

La dinámica de la relación entre el valor de la opción y el precio del activo subyacente se recoge en una ecuación diferencial parcial que refleja las tasas de cambio simultáneas de diferentes variables. Lo que hoy se conoce como la fórmula o el modelo de Black \& Scholes fue una solución publicada en 1973. Al mismo tiempo Merton publicaba un trabajo en el cual se desarrollaba un modelo mucho más generalizado para la valoración de opciones. Los trabajos en torno a la valoración de opciones financieras llevaron a Merton y Scholes a obtener el Premio Mobel de Economía en 1997. Fisher Black murió en 1995.

El modelo desarrollado por Black \& Scholes, resuelve el problema de valoración de opciones europeas (se ejercen al vencimiento) considerando los siguientes factores:

Tiempo hasta el vencimiento:

$t$

Tasa libre de riesgo:

Precio de ejercicio de la opción:

$R_{f}$

Varianza de la tasa de rentabilidad en

un instante de tiempo:
$M\left(d_{1}\right)$ : Función de distribución de la variable aleatoria normal de media nula y desviación estándar unitaria (probabilidad de que dicha variable sea menor o igual que $\mathbf{d}_{\mathbf{i}}$ ).

El propósito del modelo es determinar la relación existente entre el valor de la opción de compra (C) y el valor de la acción subyacente (So).

$$
C=S_{0} \cdot N\left(d_{1}\right)-E \cdot e^{\left(-R_{f} t\right)} \cdot N\left(d_{2}\right)
$$

Como ya se ha visto, el precio de una opción tipo call, depende de varios factores a los que de manera análoga se podría hacer referencia en un proyecto de inversión. Dichos factores son los siguientes:

- Precio de ejercicio de la opción.

- Precio del activo subyacente.

- Período de maduración / Lapso hasta el vencimiento.

- Varianza de los rendimientos.

- Tasa libre de riesgo.

A manera de ejemplo, se ilustra el uso del modelo de Black \& Scholes para calcular el valor de una opción de abandono.

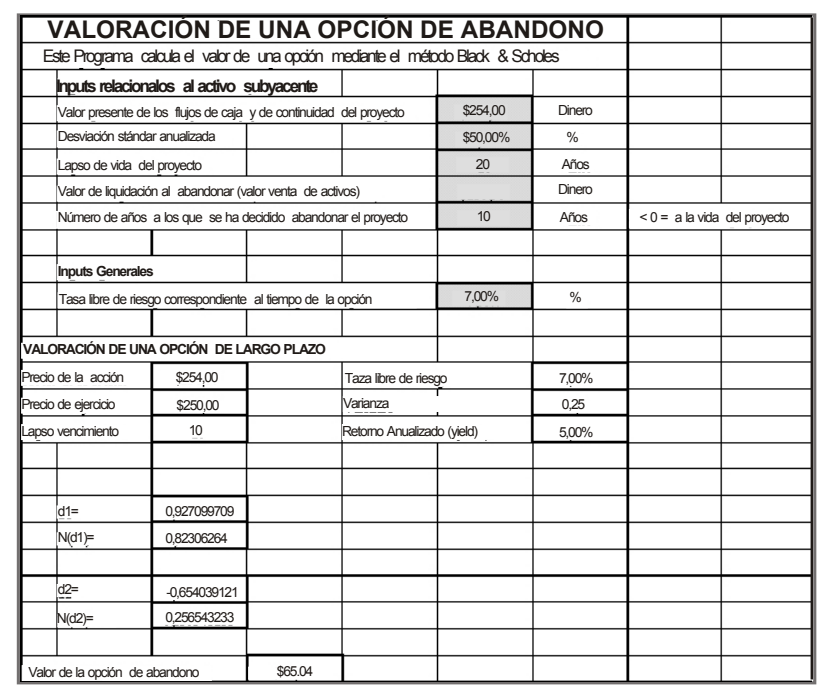

3 Mo es propósito de este artículo mostrar cómo surge la fórmula de Black \& Scholes. Los lectores interesados en esta demostración pueden consultar las referencias al final del artículo, concretamente The Pricing of Options and Corporate Liabilities Journal of Political Economy 


\section{Los proyectos de inversión como opciones tipo call:}

Cuando se analiza un proyecto de inversión con la metodología tradicional de análisis de flujo de caja, la lógica sugiere que el inversionista toma la decisión de invertir o no hacerlo y espera a ver qué sucede, es decir, espera los resultados de su decisión (Figura 3). En algunos proyectos, esta forma de actuar puede ser correcta, pero en otros proyectos, la decisión de invertir o no hacerlo puede ser consecuencia del desarrollo de ciertos acontecimientos durante la fase de evaluación del proyecto. Esta forma de actuar intenta resolver algunas de las fuentes de incertidumbre asociadas a un proyecto (Figura 3).

La gran mayoría de los proyectos de inversión que adelantan las empresas, no se pueden encajar exclusivamente en estos arquetipos. Generalmente, dichos proyectos contienen elementos, que deben ser tratados con el análisis de flujos de caja y con el análisis de opciones. Usualmente, un proyecto de inversión incorpora la necesidad de adquirir o invertir en activos reales, cuyo propósito básico es la generación de flujos de efectivo y por lo tanto el análisis de flujo de caja es pertinente como herramienta de valoración. Pero al mismo tiempo, los proyectos de inversión incorporan opciones de crecimiento, oportunidades de realizar futuras inversiones, oportunidades de abandonar algunas inversiones, oportunidades de aplazar algunas inversiones por mencionar sólo algunos ejemplos. En estos casos, el análisis de flujo de caja descontado no produce buenos resultados, por lo que se hace pertinente realizar un análisis de opciones reales. En ocasiones separar estos dos componentes es muy difícil por la naturaleza misma de algunos proyectos.

Para resolver el problema de los proyectos de inversión analizados como opciones, es necesario recurrir a una cierta analogía con las opciones financieras, particularmente sobre acciones. Un proyecto de inversión implica el derecho, pero no la obligación de adquirir una serie de activos, desembolsando una cierta suma de dinero y en un cierto periodo de tiempo.

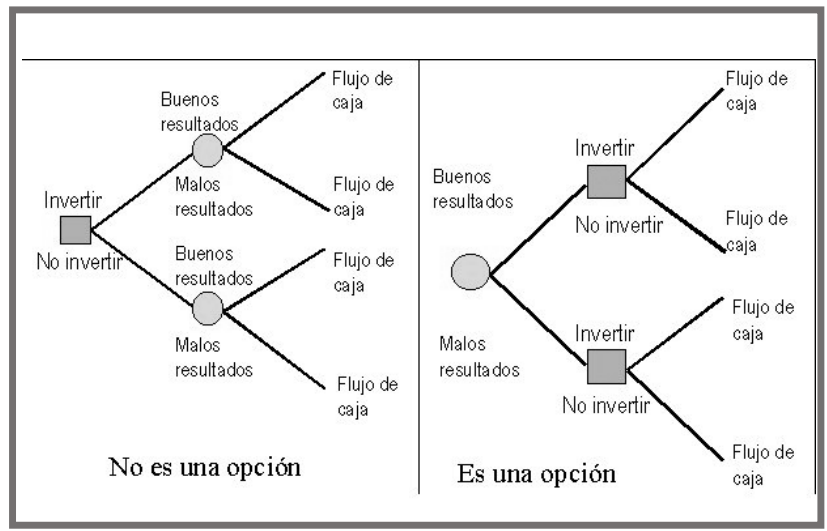

Figura 3. Caracterización de Inversiones y opciones

Es posible, entonces, plantear algunas relaciones entre los factores determinantes del valor de una opción tipo call (opción de compra) y los elementos relevantes de un proyecto de inversión.

La mayoría de los proyectos de inversión involucran decisiones en torno a la compra o construcción de activos de operación. Esto podría considerarse análogo a ejercer una opción tipo call. La cantidad invertida es el precio de ejercicio de la opción (strike price) y el valor del activo construido o adquirido es el precio del activo subyacente (precio de la acción). El lapso de tiempo durante el cual la compañía puede esperar sin perder la oportunidad de inversión es análogo al tiempo de maduración de la opción. El riesgo del proyecto de inversión se ve reflejado por la desviación estándar de los retornos de los activos. El valor del dinero en el tiempo está representado por la tasa libre de riesgo. (Figura 4). 


\begin{tabular}{|c|c|c|}
\hline Projecto & Variable & Qpaón de Compra (Call Option) \\
\hline $\begin{array}{l}\text { Desembolsos requeridos } \\
\text { para adquini el activo }\end{array}$ & $x$ & Precio de ejercicio \\
\hline $\begin{array}{l}\text { Valor de los activos operativos } \\
\text { que se van a adquinir }\end{array}$ & $\mathbf{s}$ & Precio de la acción \\
\hline $\begin{array}{l}\text { Lapso de tempo que se puede } \\
\text { demorar la decisón de } \\
\text { imiersión }\end{array}$ & $\mathbf{t}$ & $\begin{array}{l}\text { Periodo de maduración } \\
\text { Tiempo al wencimiento }\end{array}$ \\
\hline $\begin{array}{l}\text { Riesgo del activo operativo } \\
\text { subyacente }\end{array}$ & $\sigma^{2}$ & Varianza de los rendimientos \\
\hline Valor del dinero en el tiempo & $\mathbf{r}_{\mathrm{f}}$ & Tasa libre de riesgo \\
\hline
\end{tabular}

Figura 4. Proyectos vs. Opción de compra

Para analizar la relación existente entre la valoración de opciones y el análisis de flujo de caja de un proyecto de inversión, se hará uso del criterio de decisión conocido como valor presente neto (VPM). EI VPM de un proyecto no es más que la riqueza económica que el proyecto es capaz de generar. Implícitamente un proyecto con VPM positivo, incorpora el pago de las inversiones requeridas en el proyecto, el costo financiero del dinero asociado a dichas inversiones y el remanente o utilidad económica generado por el proyecto. EI VPM de un proyecto puede definirse entonces mediante la siguiente expresión:

$$
V P N(\text { proyecto })=V P(\text { Ingresos })-V P(\text { Egresos })^{4}
$$

El criterio de aceptación de un proyecto utilizando el VPM define como viables aquellos proyectos cuyo

$$
\begin{gathered}
\text { VPM }>0 \text {, es decir, } \\
\text { VP(Ingresos) }>\text { VP (Egresos) }
\end{gathered}
$$

EI VPM de un proyecto puede expresarse también como un cociente entre el VP(ingresos) y el VP(Egresos). En este caso la regla de decisión, consideraría como viables aquellos proyectos cuyo VPMa $>1$.

La figura 5, ilustra la regla de decisión para proyectos de inversión basados en el VPM calculado como cociente.

${ }^{4}$ VP se refiere a valor presente

\section{$V P N q=\frac{V P(\text { Ingresos })}{V P(\text { Egresos })}$}

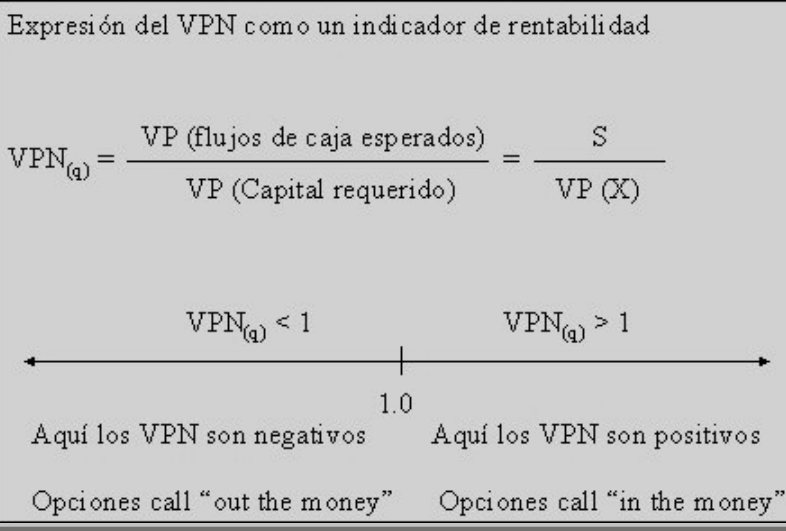

Figura 5. VPM como indicador de rentabilidad

De la observación de la figura 5, se puede inferir una regla de decisión para evaluar proyectos de inversión y en forma similar decidir si una opción call se ejerce o no se ejerce. $\mathbf{s}$ corresponde al valor del activo subyacente (precio de la acción), el cual es equivalente al VP(Ingresos o flujos de caja esperados) y $\boldsymbol{x}$ corresponde al precio de ejercicio de la opción. En forma análoga, hace referencia al VP(Egresos e inversiones del proyecto). Si VPMq $<1$, se dice que la opción está "out the money" y por lo tanto no se debe ejercer la opción (recordemos la figura 1). En caso contrario, VPMa $>1$, se debe ejercer la opción y se dice que se encuentra "in the money".

EI VPMa como indicador de rentabilidad incorpora cuatro de los elementos determinantes del valor de una opción: el precio de ejercicio, el precio del activo subyacente, la tasa libre de riesgo para descuento de los flujos y el período de maduración de la opción (t). Se puede observar que el valor de una opción call se incrementa en la medida en que crece el VPMa. 
Cuando una decisión de inversión no puede aplazarse, la opción de compra y el proyecto análogo pueden ser evaluados utilizando el análisis de valor presente de los flujos de caja. En caso contrario, si no existiese la posibilidad de aplazamiento nos encontraríamos frente a un proyecto o una opción que no ha llegado al vencimiento, por lo tanto el análisis del VPMa sigue siendo muy importante. Hasta el momento no se ha realizado ninguna referencia al riesgo inherente en el proyecto. En los siguientes párrafos se analizará el impacto del riesgo medido mediante la varianza de los rendimientos por unidad de tiempo $\left(\sigma^{2}\right)$. Si se multiplica la varianza de los rendimientos por el número de períodos de tiempo hasta el vencimiento, se obtendría la varianza acumulada $\left(\sigma^{2} t\right)$, que se constituye en una medida indicativa de la variación de los acontecimientos (rentabilidad) antes de finalizar el horizonte de tiempo a lo largo del cual se puede tomar la decisión de invertir. Mientras mayor sea la varianza acumulada, mayor será el valor de la opción (Figura 6).

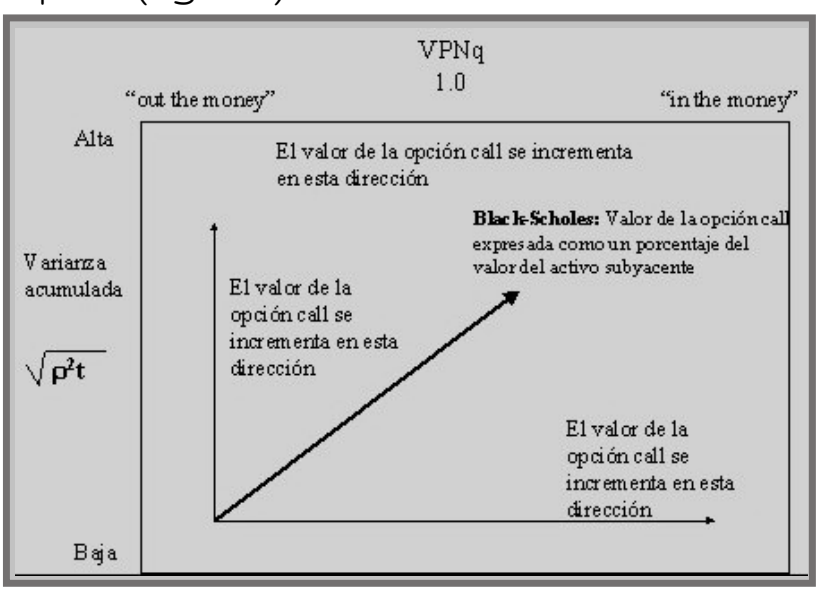

Figura 6. Valor de una opción (Varianza acumulada y VPMq)

El profesor de finanzas, Timothy Luehrman introduce una dimensión extra en el análisis de opciones reales $\left(\sigma \mathrm{t}^{1 / 2}\right)$, es decir, la raíz cuadrada de la varianza acumulada. Las opciones o proyectos para los cuales $\sigma$ ó $t$ son cero no tienen varianza acumulada y por lo tanto podrían ser evaluados con el VPM tradicional o con el VPMa.

${ }^{5}$ Capital Projects as Real Options - Luehrman Timothy
En la gran mayoría de los casos tanto $(\sigma)$ como $(t)$ toman un valor, considerado como representativo del riesgo al cual está expuesto el proyecto, por lo que el análisis tradicional por VPY pierde poder decisorio. En otras palabras, mientras más alto sea el nivel de riesgo y la incertidumbre asociada a un proyecto, los criterios de decisión tradicionales son menos útiles para explicar el comportamiento de dicho proyecto. Un ejemplo claro de esta afirmación es el crecimiento desmesurado que tuvieron las cotizaciones de las empresas de internet aún sin generar en muchos casos un solo peso de dividendos. La figura 7 ilustra en forma clara los diferentes casos que podrían presentarse al analizar una decisión de inversión considerando el valor de su VPMa, su VPM y su varianza acumulada $\left(\sigma^{2} \mathrm{t}\right)$.

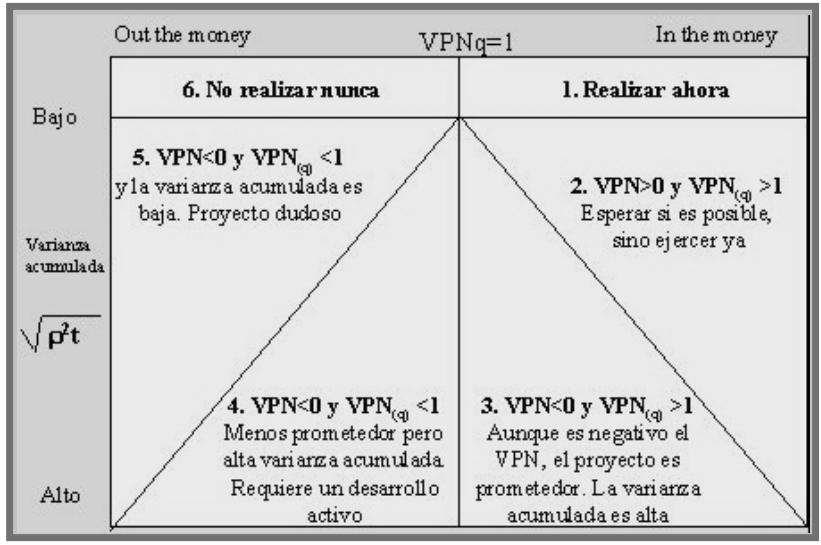

Figura 7. Mapa de decisiones frente a un proyecto o una opción

Para entender en detalle la figura 7 se revisará cada una de las zonas de decisión que podrían presentarse frente a una opción de compra o un proyecto de inversión.

Empezando por la zona 1 , se encuentran proyectos con un VPM positivo, pero con una varianza acumulada muy baja, debido a la poca variabilidad en el valor del activo subyacente o a un vencimiento muy cercano. En este caso, lo ideal es realizar el proyecto, puesto que no existen beneficios marginales por aplazarlo. 
Para entender en detalle la figura 7 se revisará cada una de las zonas de decisión que podrían presentarse frente a una opción de compra o un proyecto de inversión.

Empezando por la zona 1, se encuentran proyectos con un VPM positivo, pero con una varianza acumulada muy baja, debido a la poca variabilidad en el valor del activo subyacente o a un vencimiento muy cercano. En este caso, lo ideal es realizar el proyecto, puesto que no existen beneficios marginales por aplazarlo.

En la zona 2 la varianza acumulada se incrementa con relación a la zona 1, por lo cual, lo ideal es aplazar la decisión, ya que es probable que el valor de los activos subyacentes aumente con el tiempo. Obviamente, existe un riesgo asociado y adverso a la decisión de esperar y es una posible disminución de valor del activo. Esta situación podría ser motivada por la aparición de nuevos competidores que en la pugna por el mercado conduzcan hacia una disminución de los margenes de rentabilidad.

En la zona 3, se incluyen los proyectos que poseyendo un VPM negativo, tienen la posibilidad de obtener un VPMa positivo además de una varianza acumulada muy alta. En estos casos al ser VPMq > 1 lo más indicado es esperar y verificar el comportamiento del valor del activo subyacente. Si el valor del activo no cambia con el tiempo, la consecuencia lógica sería no ejercer la opción, es decir, no realizar el proyecto, pero en otros casos el desarrollo de los acontecimientos puede conducir a que el proyecto sea interesante. Considerando que los resultados en esta zona podrían no ser muy claros para el lector, se hará uso de un ejemplo hipotético.

Mos encontramos ante la posibilidad de realizar un proyecto que demanda una inversión inicial de un millón de pesos, que bien podría realizarse hoy o aplazarse hasta tres (3) años sin alterar la cantidad. El valor presente de los flujos de caja que se espera genere este proyecto es de
900.000 pesos. La desviación estándar de los rendimientos sobre el valor presente de los flujos de caja se estima en un $40 \%$. La tasa de interés libre de riesgo será del 5\%.

Al analizar el proyecto mediante el VPM se obtiene:

VPM $=900.000-1.000 .000=-100.000<0$ (Proyecto no viable)

Como se deduce del enunciado del ejemplo, la empresa podría aplazar la inversión inicial sin que esto alterara el monto de la misma, manteniendo el mismo valor presente para los flujos de ingreso (valor del activo). Al calcular el VPMa en la fecha de vencimiento de la opción de aplazamiento se tiene:

$$
\text { VPMa }=\frac{900.000}{1.000 .000(1.05)^{-3}}=1.0418
$$

La varianza acumulada es igual a

$\left(\sigma^{2} t\right)=(0.4)(0.4)(3)=0.48$

De acuerdo con la valoración derivada de la expresión de Black \& Scholes una opción con las características del ejemplo toma un valor del $28.8 \%$ del activo subyacente, es decir, $0.288 \times 900.000=259.200$ pesos.

EIVPM total de la inversión sería igual a :

VPM total = VPM básico + VPM opción de aplazar VPM total $=-100.000+259.200=159.200$ pesos

Los resultados anteriores significan que si el proyecto se realiza hoy como estaba inicialmente previsto, el VPM lo señalaría como un proyecto no viable, pero si se aplaza la decisión hasta un máximo de tres años es muy probable que las condiciones cambien favorablemente para el proyecto o la opción. Lo que realmente se espera en este proyecto es que el valor del activo subyacente se incremente a una tasa superior a la tasa de libre de riesgo. 
Se reconoce que en un entorno económico cambiante como el actual, es imposible diferir en el largo plazo las inversiones de un proyecto sin que se presenten modificaciones en la magnitud de las mismas. Sin embargo, debe observarse que la opción de aplazar sigue mejorando el VPM del proyecto aún permitiendo un cierto crecimiento en el monto a invertir, en virtud del aplazamiento.

En la zona 4, aparecen proyectos con VPM negativo, con VPMa $<1$, pero con alta varianza acumulada, que podría resultar favorable, si se demorase la realización del proyecto esperando que mejore el valor presente de los flujos del proyecto o el valor del activo subyacente.

En la zona 5, las condiciones son muy similares a la zona anterior, con la diferencia de que la variabilidad del activo subyacente es menor, por lo que existen pocas posibilidades de viabilidad económica para el proyecto.

En último lugar, en la zona 6 se encuentran aquellos proyectos cuyos indicadores convencionales (VPM y VPMq) son desfavorables y no presentan ningún tipo de variabilidad en los resultados (carencia de riesgo), lo que significa que estos proyectos no se deben realizar bajo ninguna razón.

Para reforzar los resultados anteriores, conviene señalar que en la medida en que el tiempo transcurra, la incertidumbre (variabilidad) irá disminuyendo, aumentando las posibilidades de éxito del proyecto.

\section{CONCLUSIONES}

- El método de las opciones reales se constituye en una excelente herramienta para el análisis de proyectos de inversión en ambientes de incertidumbre. Esta metodología, no reemplaza el análisis tradicional por flujos de caja, pero lo complementa en aquellos casos en donde el valor presente neto (VPM) subvalora proyectos de inversión que incorporan consideraciones de carácter estratégico.
- El análisis de opciones reales no invalida el uso de herramientas estadísticas, por el contrario hace uso intensivo de las mismas. Los problemas que se presentan al utilizar los métodos de valoración de opciones financieras sobre activos reales se intentan reducir mediante el uso de técnicas como la simulación (Montecarlo) a fin de determinar los múltiples valores que podría tomar el activo subyacente (proyecto).

- La utilización reiterada del método de Black \& Schooles para valorar opciones reales presenta el inconveniente de que algunos proyectos no son fácilmente replicables mediante un portafolio de títulos financieros en mercados bursátiles, condición básica del modelo sugerido por estos autores. La presencia de portafolios replicables en mercados de valores eficientes se convierte en un patrón del riesgo financiero que algunos proyectos podrían tener.

- El análisis mediante opciones reales permite integrar la teoría financiera tradicional con el análisis y la planeación estratégica, permitiendo una valoración más apropiada de aquellos proyectos con alto nivel de incertidumbre, aunque debe apoyarse en herramientas estadísticas que simplifiquen algunos de los problemas presentes en su aplicación.

- En torno al análisis de opciones reales se pueden configurar dos grandes áreas de investigación en el presente y futuro. La primera de ellas tiene que ver con el incremento del número de aplicaciones empresariales de la metodología (proyectos de investigación y desarrollo, proyectos de innovación tecnológica, proyectos de inversión en sistemas de información, etc.). La segunda, hace referencia al mejoramiento de las técnicas de valoración de opciones reales, incorporando elementos como árboles de decisión o simulación, áreas con un gran potencial de investigación. 
- El análisis de proyectos mediante opciones reales ha generado enormes expectativas en el ambiente académico, pero el ritmo de penetración de esta metodología en el ambiente empresarial ha sido más lento de lo que se esperaba, debido principalmente a que la introducción del método se ha centrado en torno a los aspectos técnicos y matemáticos de los modelos de valoración, sin considerar que esta metodología constituye en sí misma una nueva forma de afrontar las decisiones de inversión.

- Las organizaciones al realizar el análisis de sus alternativas de inversión deberían clasificar los proyectos de acuerdo con las opciones reales que se incluyen. La clasificación de acuerdo con los criterios tradicionales de reemplazo de equipos, reducción de costos, aumento de la capacidad, resulta de poca utilidad frente a una diferenciación de los proyectos cuyos beneficios se obtienen mediante flujos de caja y aquellos cuyos beneficios se derivan de opciones sobre decisiones posteriores. Las empresas deben clasificar los proyectos entre aquellos que requieren inversión inmediata y aquellos sobre los que se tiene flexibilidad para decidir en el futuro.

- El análisis mediante opciones reales muestra que la incertidumbre, las tasas de interés más altas y los horizontes de tiempo más lejanos no son necesariamente perjudiciales para un proyecto. A pesar de que estas variables reducen el valor presente neto estático del proyecto, también pueder provocar un aumento en el valor de las opciones asociadas al proyecto que puede contrarrestar el efecto negativo sobre el valor presente.

\section{BIBLIOGRAFÍA}

AMRAM, M., y M. KULATILAKA, Real options, Harvard Business Press, Boston Mass., 1999.

BLACK, FISHER y SCHOLES, Myron. The Princing of Options and Corporate Liabilities. Journal of Political Economy. Mo. 81. Mayo ñ Junio de 1973. Pp. 637-659.
CARR, P.," The Valuation of Sequential Exchange Opportunities", The Journal of Finance, Vol. 43, pp. 1235-1256, Dec. 1988.

COURTMEY, H., J. KIRKLAND, and P. VIGUERIE, "Strategy Under Uncertainty", Harvard Business Review, pp. 67-79, Mov.-Dec. 1997.

DIXIT, a.k., and R.S. PIMDYCK, "The options Approach to Capital Invesment," Harvard Business Review, pp. 105-115, May-June 1995.

HERATH, H.S.B., and C.S PARK, "Economic Analysis of R\&D Projects: An Options Approach", The Engineering Economist, Vol. 44 Mo. 1, Pp. 135, 1999 .

LUEHRMAM, T.A., "What's It Worth: A General Managers Guide To Valuation," Harvard Business Review, pp. 105-115, May-June 1997.

LUEHRMAM, T.A., "Investment Opportunities as Real-options: Getting Started on the Numbers, Harvard Business Review, July-Aug., PP. 51-67, 1998.

LUEHRMAM, T.A., "Strategy as a Portafolio of Realoptions", Harvard Business Review, Pp. 88-99, Sept-Oct. 1998

ROSS, S.A., "Uses, Abuses, and Alternatives to the Met-Present-Value Rule," Financial Management, Vol. 24, Mo. 3, Pp. 96-102, Autumn 1995.

SHARP, D.J., "Uncovering The Hidden Value in High Risk Investments", Sloan Management Review, pp. 69-74, Summer 1991.

SMITH, J., and R. MAU, "Valuing Risky Projects: Option Pricing Theory and Decision Analysis", Management Science, Vol.41 Mo. 2 pp. 795-816, Apr. 1995.

TRIGEORGIS, L., "Real Options and Interactions with Financial Flexibility", Financial Management, Vol. 22 Mo. 3, pp. 202-224, 1993.

TRIGEORGIS, L., "The Mature of Option Interactions and the Valuation Of Investments with Multiple Real Options," Journal of Financial and Quantitative Analysis, Vol. 28 Mo. 1, pp. 120, Mar. 1993. 